\title{
La "dromocracia" o el régimen de la velocidad absoluta (Paul Virilio). Un diagnóstico de sus derivaciones mórbidas en la existencia.
}

\section{"Dromocracy" or the Regime of Absolute Speed (Paul Virilio). A Diagnosis of its Morbid Implications on Existence.}

\author{
Borja García Ferrer ${ }^{1}$ \\ Universidad de Granada (España)
}

Recibido: 24-04-16

Aprobado: 02-05-16

\section{Resumen}

Este trabajo comprende dos grandes bloques. En el primero, examinamos en perspectiva histórica, tomando como referencia la figura de Paul Virilio, el impacto político de la velocidad. Tras jugar un papel fundamental en todas las épocas, el poder totalitario y tecnológico del presente pivota, según el autor francés, sobre el extraordinario incremento de la velocidad que comportan las nuevas tecnologías de la telecomunicaión, con menoscabo del concepto tradicional de espacio y de la idea misma de Estado. En el segundo bloque, probamos el alcance de la crítica viriliana en el terreno de la crítica de patologías, en lo que constituye la aportación más original del artículo. Según nuestra hipótesis, existe una discrepancia patógena entre la lógica de la aceleración que funda la infoesfera y el funcionamiento de los receptores humanos, en la medida que nos despoja, por razón de cierta "presentificación" del tiempo real, de los tiempos

\footnotetext{
${ }^{1}$ (borja_co@hotmail.com). Doctor en Filosofía por la Universidad de Granada. Sus intereses de investigación actuales son la filosofía contemporánea, la ontología política, la sociología crítica y la psicopatología. Entre sus últimas publicaciones, destacan: "La comunidad ortopédica. Por una crítica de patologías desde el paradigma inmunológico", Isegoría. Revista de Filosofía Moral y Politica, 52 (2015), pp. 331-348. "La constitución de la identidad personal en el nuevo capitalismo y sus repercusiones morales", Daimon. Revista Internacional de Filosofia, 67 (2016), pp. 117131. "Revolución espacial y 'desarraigo'. Una crítica de sus implicaciones psico-somáticas en el capitalismo virtual”, Pensamiento. Revista de Investigación e Información Filosófica, 72, 274 (2016), pp. 1175-1196. "La 'agenesia' como patología de civilización y su alcance en el capitalismo de la flexibilidad”, Co-herencia, 14, 26 (2017), pp. 119-150.
} 
de elaboración necesarios para atender sus demandas de movilidad. De aquí se siguen "enfermedades de la adaptación" tan inquietantes como extendidas, como atestigua fehacientemente la ciencia empírica.

Palabras-clave: Virilio, Infoesfera, Velocidad, Espacio, Tiempo, Patología.

\begin{abstract}
This work includes two great blocks. In the first one, we examine the political impact of speed from a historical point of view, taking the figure of Paul Virilio as a reference. Having played an essential role in the past, the totalitarian and technological power of present times revolves around the extraordinary rise of speed brought about by the new telecommunication technologies, to the detriment of the traditional concept of space and the very idea of State. In the second block, we test the scope of Virilio's criticism in the field of the criticism of pathologies, which is the most original contribution of this work. According to our hypothesis, there exists a pathogenic discrepancy between the logic of acceleration founded by the infosphere and the ways of the human beings, in as much as it deprives us, due to a certain "presentification" of real time, of the processing times necessary to attend our mobility demands. Hence it follows a series of widespread and disturbing "adaptation diseases" as demonstrated irrefutably by empirical science.
\end{abstract}

Key-words: Virilio, Infosphere, Speed, Space, Time, Pathology.

El Tiempo y el Espacio murieron ayer. Ahora vivimos en lo absoluto, puesto que ya hemos creado la velocidad omnipotente

F. T. MARINETTI, Manifiesto del futurismo

\title{
1. El régimen de la velocidad absoluta en perspectiva histórica
}

A pesar de su indiscutible sagacidad, ninguna versión de la historia oficial ha reparado lo suficiente, con la notable excepción de Virilio, en la misiva de Goebbels cuando identifica la calle, en el marco del conflicto entre nacionalsocialistas y marxistas (Berlín, 1931), con la arena política. Y es que, si lanzamos una mirada atenta a la "intrahistoria" (Unamuno), es fácil reparar en el factum de que el contingente revolucionario alcanza su forma ideal en algo que escapa a titulares de prensa, debates científicos y tertulias intelectuales, a saber, la muchedumbre de caminantes anónimos (“dromómanos", en términos 
psiquiátricos) que deambulan erráticos, sin rumbo fijo, a la deriva. Toda revolución, es cierto, hunde sus raíces en ese vagabundeo invisible por la red de trayectorias que constituye la calle, un primer transporte común donde la "libertad de ir y venir" (Montaigne) termina configurando, en cuanto productor de velocidad, una "máquina de asalto" cuyo movimiento es asimilado al progreso hacia una tierra ignota. Se entiende, desde este prisma, por qué la esencia de la velocidad no es, para Virilio, sino el poder: "Poder y velocidad son inseparables al igual que riqueza y velocidad son inseparables... Poder es siempre poder de controlar un territorio con mensajes, modos de transporte y comunicación. (...) Un acercamiento a la política es imposible sin un acercamiento a la economía de la velocidad"2.

Para controlar el movimiento de las masas y contener su inercia revolucionaria, el poder ha seguido invariablemente un esquema políticopolicial, transformando los centros populares de circulación intensa y transporte rápido en "grandes máquinas inmóviles diversamente fabricadas" (Vauban), desde las fortificaciones rurales galorromanas hasta las fortalezas medievales puramente militares. Desde esta perspectiva, la fuerza de la burguesía, por ejemplo, no estribaba tanto en el comercio como en la implantación estratégica del "domicilio fijo" como valor monetario y social, ese "derecho" a residir allende la muralla de la ciudad fortificada que permite prever los desbordes del flujo social, implantando toda clase de filtros en aras de disuadir a la masa móvil de la "tentación" de la calle: "Las antiguas playas pantanosas y malsanas que rodeaban la ciudad fortificada, las viejas fortificaciones, las zonas, las villas miseria y favelas pero también el hospicio, el cuartel, la prisión, no resuelven tanto un problema de encierro o de aislamiento como un problema de circulación; todos son lugares inciertos porque están entre dos velocidades de tránsito, actuando como frenos a la penetración, a su aceleración”’3.

\footnotetext{
${ }^{2}$ Paul Virilio, El cibermundo. La política de lo peor, Madrid, Cátedra, 1997, p. 15. En contraste con Marx y Foucault, Virilio antepone el poder de la velocidad al poder que representan la economía o el conocimiento, sin dejar de reconocer la importancia de éstos. Cfr. Ibíd., p. 59. De hecho, el filósofo francés puede presumir de protagonizar un acercamiento único a la ciencia política, en ruptura con los presupuestos del positivismo científico que han guiado la teoría y la praxis desde el siglo XVIII, pero también con las diversas corrientes antipositivistas (marxista, freudiana, constructivista, postmoderna, feminista, etc.) aparecidas a partir de los años sesenta en el terreno de la ciencia política con el nombre genérico de "Perestroika". Aunque muchos de sus contemporáneos inscriben su filosofía en el postmodernismo, Virilio se ha desmarcado en repetidas ocasiones del llamado "pensamiento débil" para abrazar la corriente fenomenológica, en virtud de la profunda admiración que siente por Husserl. Cfr. Paul Virilio, Ground Zero, Londres, Verso, 2002, p. 153.

${ }_{3}^{3}$ Paul Virilio, Velocidad y política, Buenos Aires, La Marca, 2006, p. 17. A lo largo de su prolija obra, Virilio utiliza indistintamente los dos conceptos básicos de la cinemática, a saber, "velocidad" [vitesse] y "aceleración", bajo el supuesto de que los físicos denominan "velocidad positiva" y "velocidad negativa", respectivamente, a los procesos de "aceleración" y "desaceleración". Cfr. Paul Virilio, La vitesse de libération, París, Galilée, 1995, pp. 16, 24, 45, 47, 172. Sin embargo, Sokal y Brickmont han señalado a propósito, con el objeto de ilustrar el supuesto carácter pseudocientífico de la filosofía de Virilio, que la aceleración se define propiamente, en términos de la ciencia física, como "tasa de variación" de la velocidad. Cfr. Alan Sokal y Jean Brickmont, Imposturas intelectuales, Barcelona, Paidós, 1999, pp. 169-170.
} 
No obstante, la revolución burguesa extiende el estado de sitio de la máquina inmóvil, como novedad fundamental, al "vasto campo de la nación" (Barère), de manera que el poder estatal y el orden social se truecan, respectivamente, en vialidad y control de la circulación, por medio de mecanismos como el alojamiento social en ciudades dormitorio, los sistemas de peaje en las autopistas o los cuarteles generales de la gendarmería, por citar algunos ejemplos de la miríada de órganos encargados de administrar la fortaleza urbana según el nuevo proyecto logístico del poder, etiquetado como "Defensa nacional" por la revolución burguesa en su particular afán por volver transparente el espacio humano a la mirada policial. De este modo, el ingeniero militar asume, elevado como "sacerdote de la civilización" (Saint-Simon), los galones del "castrámeta", complementando su función militar con una especie de geometría descriptiva de la naturaleza donde la finalidad de la fortificación no es tanto contener los ejércitos como favorecer su movimiento pues, como saben los seguidores de Trotsky, el teórico de la "revolución permanente", la defensa del territorio depende de la transformación para ser efectivo y, por ende, el arte de la guerra debe ser "estratégico", es decir, conjugar la ofensa y la defensa; en palabras de un estratega chino: "Un ejército es cada vez más fuerte cuando puede ir y venir, extenderse y replegarse, como quiere y cuando quiere".

Desde este punto de vista, la Revolución Francesa designa, del movimiento sans-culotte al "reclutamiento masivo" del 93, una organización racional de los flujos de circulación, un secuestro de las masas nómadas por concurso del cual las fuerzas populares parisinas emprenden, erigidas en agentes logísticos de la "policía", un asedio continuo al ritmo de "la Marsellesa". De tal suerte que, en lo sucesivo, la fuerza de los ejércitos se cifra, parafraseando a Napoleón I, en su "masa multiplicada por su velocidad", toda vez que la nueva dialéctica del campo de batalla introduce al soldado en la trayectoria de los vehículos balísticos, de forma que la salvación general estriba inexorablemente en el viejo "correr hacia delante". Se trata, en efecto, de la primera dictadura del movimiento, cuya capitalización sustituye el viejo sometimiento a la coacción indeterminada y el encierro arbitrario.

Con el fracaso de la ciudadela burguesa, consumado por la desaceleración de la guerra civil hacia la colisión urbana, los regímenes totalitarios patrocinan, con independencia de su ideología, la evolución del "Estado-máquina" al "planeta-máquina", por medio de una continuación "policial" a mayor velocidad, en diferentes vehículos. Para aumentar el dominio estatal sobre la circulación de las masas, el totalitarismo pone el acento en los ejércitos y las policías, desarrollando plenamente la energía cinética de la masa revolucionaria, es decir, su aptitud para el movimiento, como ilustran las fiestas totalitarias donde grandes conjuntos geométricos integran el dinamismo de los individuos en una suerte de decoración caleidoscópica. En este sentido, asimila la velocidad del 
asalto y la invasión, como ilustra la "guerra relámpago" nacionalsocialista, a la mecánica de una revolución, de modo que el objeto de la guerra se cifra en llevar las fronteras nacionales al territorio del otro siguiendo las consignas fundamentales de Clausewitz y Napoleón, o sea, por la vía rápida, según la declamación dinámica del caudillo de turno.

En cualquier caso, el auge del totalitarismo es inseparable de la revolución de los transportes (vale decir, de las máquinas de destrucción), como pone de manifiesto la tragedia fascista. Con la superación técnica de la plaza fuerte, termina el violento despotismo que aspiraba, bajo la égida de los monopolios comerciales, a la dominación exclusiva de la explanada marítima, convirtiendo los océanos en un vasto campo logístico. Pues bien, lejos de significar el ocaso definitivo de las relaciones de dominio, cuando el transporte abandona el elemento marino por efecto del progreso tecnológico tiene lugar un cambio de velocidad de la economía mundial sin precedentes, por mor del cual los Estados modernos se tornan totalitarios, en detrimento de las naciones cuya superioridad económica venía consagrada por la práctica del mar. En efecto, la lógica del poder/mover expande su dominio sobre todas las superficies del planeta. Hemos llegado a la "guerra total".

Tras abandonar la explanada de las trincheras, las masas recuperan su libertad de movimiento y la batalla política por construir el aparato de guerra con la mejor eficacia dinámica posible emprende, con la apoteosis de la sociedad de consumo, el desarrollo de la industria de la comunicación y la proliferación del american life style como telón de fondo, un cambio de rumbo decisivo. En este punto del proceso "dromológico"4, el objetivo del poder se cifra en superar definitivamente los problemas creados por el acondicionamiento militar de los territorios en la época de las máquinas totalitarias, hasta el prurito de llevar a cabo una guerra sin aquí, allende las hazañas logradas por el auto blindado todo terreno o las aeronaves de la Luftwaffe alemana, presentadas a la humanidad por el capitán de Poix y el mariscal Göring en 1915 y 1939, respectivamente.

En el empeño por superar la crisis económica de los años treinta mientras disuade a las masas de su tendencia a la revolución, los Estados Unidos depositan toda su confianza en la capacidad de transporte que supone la producción de automóviles en cadena, al hilo de la intuición fundamental de Helmut Klotz en 1937 sobre la importancia de la motorización para aumentar exponencialmente el poder de las Fuerzas de Asalto nacionalsocialistas. Sin embargo, si bien es cierto que la estética exuberante del automóvil norteamericano apenas ocultaba sus limitaciones de velocidad aplicadas como estratagema de vialidad política, dicha prohibición vehicular siembra la semilla de un nuevo inicio, en la medida

${ }^{4}$ Con este término (del griego "dromos", esto es, "velocidad"), Virilio hace referencia a la Revolución Industrial, en aras de mostrar no sólo la aparición de la tecnología, sino la razón instrumental que obedece. 
que numerosos conductores privados de las grandes velocidades comienzan a indagar, espoleados por su profundo sentimiento de frustración, el mundo de la mecánica, en lo que constituye un entrenamiento intensivo que terminará forjando un nuevo aparato de guerra.

En efecto, la revolución microelectrónica de los 40 proporciona una geometría propicia a la velocidad de cara a cumplir de una vez por todas la promesa del movimiento, superando los obstáculos que impedían desarrollar plenamente la ubicuidad del transporte en un escenario geopolítico definido arbitrariamente como "paz total" o "equilibrio del terror", esa ilusoria coexistencia pacífica que oculta, sin declaración de hostilidades, un desequilibrio en continuo aumento. Con el advenimiento de las nuevas tecnologías de la telecomunicación, la información atraviesa el planeta de forma instantánea y los acontecimientos pueden ser compartidos virtualmente mediante su transmisión en tiempo $\mathrm{real}^{5}$, de forma que el poder registra y condensa todas las edades del mundo, desde las grandes operaciones comerciales hasta el "mundo de la vida" (Lebenswelt). Es lo que Virilio denomina "globalitarismo" o "imperio de la velocidad", una suerte de "totalitarismo de totalitarismos" de alta tecnología que funciona desmembrando los cuerpos territoriales para convertirlos en meros instrumentos del poder, de modo que resulta infinitamente más peligroso que las versiones nacionalsocialista o estalinista del totalitarismo, ya que se expande más allá del ámbito continental ${ }^{6}$.

5 Virilio observa con asombro que las noticias, las operaciones financieras, la información estratégica geopolítica, etc. llegan a transmitirse, literalmente, a la velocidad de la luz. Para explicar semejante fenómeno, el autor se inspira en la teoría de la relatividad, cuya innovación constituye, a sus ojos, una especie de revelación cultural inadvertida. Según la convención habitual en física, existen dos tipos de intervalos ("tiempo" y "espacio"), cuyas "longitudes invariantes" son positivas y negativas, respectivamente. Dichos intervalos han ordenado la geografía y la historia del mundo, para Virilio, mediante la geometrización de los ámbitos agrarios (parcelación) y urbanos (catastro), así como la organización calendaria y la medida del tiempo (relojes) han regido la regulación cronopolítica de las sociedades humanas. Pues bien, la teoría de la relatividad introduce un nuevo tipo de intervalo, a saber, el intervalo del género "luz" (velocidad-límite, signo cero), una constante cosmológica sin cuyo concurso resultan inconcebibles los intervalos de tiempo (duración) y espacio (extensión), en lo que constituye un salto cualitativo sin precedentes, una transformación radical de la relación del hombre con su medio vital. Cfr. Paul Virilio, La vitesse de libération, op. cit., p. 25. En otras palabras, "a la cronología tradicional (futuro, presente, pasado) le sucede desde ahora la CRONOSCOPIA (subexpuesto, expuesto, sobreexpuesto). El intervalo de tipo TIEMPO (signo positivo) y el intervalo de tipo ESPACIO (signo negativo) (...) sólo se inscriben gracias a la LUZ, a ese intervalo del tercer tipo, cuyo signo cero es índice de la celeridad absoluta. Cfr. Paul Virilio, TransAppearance, “Artforum", 27, 10 (1989), p. 129. Cfr. Paul Virilio, L'inertie polaire, París, Christian Bourgois, 1990, pp. 108-109, 115. Sokal y Brickmont han reprochado a Virilio, empero, su escaso rigor intelectual, ya que se trata de intervalos en el espacio-tiempo, los cuales no coinciden con lo que suele denominarse "espacio" y "tiempo"; además, no guardan ninguna relación, según ambos autores, con algo así como "la geografía y la historia del mundo" o la "regulación cronopolítica de las sociedades humanas". Cfr. Alan Sokal y Jean Brickmont, op. cit., pp. 170-171. Una interesante introducción a la noción de "espacio-tiempo" se encuentra: Edwin Taylor y John Archibald Wheeler, Spacetime Physics, San Francisco, W. H. Freeman, 1966.

${ }^{6}$ Cfr. John Armitage, From modernism to hypermodernism and beyond: an interview with Paul Virilio [en Paul Virilio, From modernism to hypermodernism and beyond, Londres, Gran Bretaña, Sage, 
Y es que el incremento de los rendimientos vehiculares no sólo renueva el poder implosivo de los vehículos subsónicos, sino que también implementa el poder destructivo de los explosivos moleculares o nucleares, arrojándonos telúricamente a un horizonte topológico definido por la negación del espacio: "En esta contracción geográfica (...) la penetración y la destrucción se confunden, la instantaneidad de la acción a distancia corresponde a (...) la derrota del mundo como campo, como distancia, como materia"7. En tal disposición de los términos, la masa planetaria se trueca repentinamente, toda vez suturada la "brecha tecnológica" que separaba y, al mismo tiempo, diferenciaba la humanidad, en un conglomerado homogéneo, una sola "interfaz" donde la localización geográfica pierde el protagonismo que disfrutaba en la guerra de las fuerzas mecanizadas a favor del "no-lugar" de la velocidad ${ }^{8}$. Entonces, la nueva máquina de guerra accede a la velocidad superior del Asalto, sin la cual riqueza y acumulación jamás habrían sido posibles; por oposición a los conflictos bélicos y políticos de la Modernidad, lo decisivo ya no es la aceleración mecánica del transporte, sino la velocidad absoluta ${ }^{9}$ en el ámbito de la información: "Desde que los objetos, las mercancías y las personas pudieron ser sustituidas por signos, por fantasmas virtuales transferibles por vía electrónica, las fronteras de la velocidad se han derrumbado y se ha desencadenado el proceso de aceleración más impresionante que la historia humana haya conocido"10.

2000], pp. 25-26. Es de recibo reconocer que asimilar el progreso tecnológico a una reactualización del totalitarismo es una tesis sumamente original, toda vez que la mayoría de los análisis sobre el desarrollo de la tecnología (es decir, aquellos que conforman el discurso dominante en la actualidad) no hacen referencia alguna a cuestiones políticas e ideológicas, como si se tratase de un proceso racional, neutral y necesario, motivado exclusivamente por razones técnicas y esencialmente inherentes al proprio progreso tecnológico. Cfr. Imanol Zubero, Los sindicatos españoles ante el cambio tecnológico (entre 1975 y 1990), Bilbao, Desclée de Brouwer, 1993. En contraste con la visión de Virilio, las nuevas tecnologías suelen presentarse, de hecho, como "tecnologías abiertas", en el sentido de que encierran infinitas potencialidades, más allá del contexto social donde se inscriben. $C f r$. Pierre-Alain, Mercier, François Plassard y Víctor Scardigli, La sociedad digital, Barcelona, Ariel, 1985. Dichas posibilidades han dado lugar a diversas utopías postindustriales conservadoras (véase Masuda, Toffler o Naisbitt), según las cuales "de la mano de las nuevas tecnologías de la información y de la comunicación surgirá la esperada liberación humana en forma de fluidez comunicativa, auto-realización personal, revalorización de lo comunitario". Ramón Zallo, La ciencia y la tecnología como relaciones sociales, “Telos", 17 (1989).

${ }^{7}$ Paul Virilio, Velocidad y política, op. cit., p. 119.

${ }^{8}$ Virilio define físicamente el espacio dromosférico (esto es, el "espacio-velocidad") mediante la llamada "ecuación logística", resultado de multiplicar la masa desplazada por la velocidad de su desplazamiento (M x V). Cfr. Paul Virilio, L'espace critique, París, Christian Bourgois, 1984, p. 176. No obstante, Sokal y Brickmont han desmentido la tesis de Virilio en los términos siguientes: "La ecuación logística es una ecuación diferencial que se estudia en la biología de las poblaciones, entre otros campos. Se escribe $\mathrm{dx} / \mathrm{dt}=\mathrm{Xx}(1-\mathrm{x})$ y fue enunciada por el matemático Verhulst (1838). No tiene nada que ver con M x V. En mecánica newtoniana, M x V se llama 'momento', y en mecánica relativista no aparece para nada". Alan Sokal y Jean Brickmont, op. cit., p. 173.

${ }^{9}$ No debemos entender la expresión "velocidad absoluta" en un sentido literal, sino para representar la increíble velocidad que ha alcanzado la tecnología en relación a la velocidad relativamente modesta que tomaba antes de la irrupción de las nuevas tecnologías de la telecomunicación.

${ }^{10}$ Franco Berardi, La fábrica de la infelicidad. Nuevas formas de trabajo y movimiento global, Madrid, Traficantes de sueños, 2003, p. 176. 
Ahora bien, el descompromiso geográfico que designa la desvalorización de las localizaciones trasciende la retirada estratégica de los ejércitos convencionales, pues el tiempo ganado por el movimiento de retroceso respecto al espacio (entendido como "campo de acción") no persigue contrarrestar la fuerza efectiva de un adversario. Se trata, en contraste con el viejo empeño por contener el movimiento en curso, de desarrollar exponencialmente la capacidad de los nuevos medios de comunicación para reducir el espacio con el efecto multiplicador de la velocidad, círculo vicioso que delega la fatalidad de la destrucción en la producción. En efecto, la dimensión totalitaria de la tecnología deriva, precisamente, de la "voluntad de aceleración" que ésta impone. El mantenimiento del monopolio imprime la necesidad perentoria de oponer uno más veloz a todo dispositivo novedoso, en una suerte de carrera tecnológica ("dromológica", en términos de Virilio) que trasciende las barreras de la velocidad, hasta el prurito de superar el sistema de ganancia de la obsolescencia industrial ${ }^{11}$. Hemos pasado, en definitiva, de la fase del fuego o del explosivo a la del movimiento de los vectores, del estado de sitio de las guerras del espacio al estado de urgencia de la "guerra cronológica", donde las maniobras técnicas para conquistar el instante sustituyen a las tácticas que buscaban invadir el terreno, como revela el abandono del canal de Panamá por parte de la soberanía norteamericana.

Así las cosas, el poder deviene una especie de "meteorología" donde cada velocidad designa un "departamento" del tiempo, en detrimento de la libertad de decisión y acción política que no hace tanto determinaba el destino de los Estados y sus alianzas, reemplazados progresivamente por la realidad virtual de los "tele-continentes" y la "ciudad global" donde se cifra el régimen de la velocidad absoluta, esa "meta-ciudad des-territorializada que se va a convertir (...) en el sitio de la metro política, cuyo carácter totalitario, o mejor, 'globalitario', será obvio a la vista de todos" ${ }^{\prime 2}$. Mientras la aceleración creciente

${ }^{11}$ Como el umbral de la velocidad disminuye sin cesar, el artefacto rápido es cada vez más complejo de concebir y, con frecuencia, está obsoleto incluso antes de ser explotado. En este sentido, la nueva carrera armamentística encuentra su razón de ser en el código artístico del modernismo que, en el afán por afirmar, más allá de cualquier variación estilística, un orden radicalmente distinto, prohíbe el estancamiento y exige la invención permanente. De este modo, destruye y desprecia las obras que instituye tan pronto como ven la luz, lo nuevo se torna ipso facto viejo, pasa a la retaguardia y se pierde en el olvido. Cfr. Gilles Lipovetsky, La era del vacío. Ensayos sobre el individualismo contemporáneo, Barcelona, Anagrama, 1990, pp. 81-85.

${ }_{12}$ Paul Virilio, Art of the motor, Minneapolis, University of Minnesota Press, 1995, p. 11. La devaluación del Estado en cuanto "organización del límite" constituye ya un tópico de la bibliografía contemporánea. Por ejemplo, Bauman sugiere que semejante fenómeno debe ser entendido a la luz del modelo del Dios tardo-medieval elaborado por los franciscanos y los nominalistas (incognoscible, impredecible e indiferente al bien y al mal). Desde este punto de vista, todos los intentos de forzar la mano de Dios estaban destinados a fracasar, ya que Él no debe nada a los hombres: Una vez consumado el acto de la creación, declaró sin muchas palabras su total indiferencia ante los asuntos humanos. Pues bien, análogamente, hoy toca al Estado seguir el ejemplo del Dios franciscano/nominalista y retirarse. Cfr. Zygmunt Bauman, La società sotto assedio, Roma, Laterza, 2007, pp. 31-32. 
de los rendimientos vehiculares extiende la miniaturización estratégica del espacio al campo político, los ingenieros encargados de su desarrollo asumen la libertad de maniobra que poseían los hombres de Estado y sus delegaciones subalternas, jefes de guerra y otros generales a la hora de concebir la estrategia más oportuna en cada momento, en espera del advenimiento inminente de un sistema tecno-científico e industrial autosuficiente; en palabras de Virilio: "Los protagonistas practican en lo sucesivo la política de lo peor o, más exactamente, la 'apolítica de lo peor', que fatalmente conduce a que la máquina de guerra se convierta un día en la decisión misma de la guerra, llevando a cabo así la perfección de su autosuficiencia, la automatización de la disuasión"13.

Se entiende, desde esta perspectiva, la misiva de Heidegger en el encuentro internacional de los premios Nobel en Lindau (1955), según la cual la audacia de la investigación científica estaba gestando, con los medios de la técnica, una transformación del mundo cuyo alcance rebasa ampliamente el peligro que representaba la bomba de hidrógeno durante la época atómica ${ }^{14}$. No obstante, lo más inquietante no era, a su juicio, la inminente tecnificación del mundo, sino que ningún individuo, grupo o comisión, ya sean eminentes hombres de estado, investigadores, técnicos o directivos de la economía y la industria, estaba preparado para afrontar semejante transformación universal, en virtud de lo cual nos encontramos abocados, tan indefensos como desconcertados, a cumplir los designios de la técnica en su irresistible prepotencia: "No sabemos por cuánto tiempo el hombre se encuentra en una situación peligrosa ¿Por qué? ¿Sólo porque podría de pronto estallar una tercera guerra mundial que tuviera como consecuencia la aniquilación completa de la tierra y la destrucción de la humanidad? NO. (...) ¡Extraña afirmación! Extraña, sin duda, pero sólo mientras no reflexionemos sobre su sentido. (...) ¿Qué gran peligro se avecinaría entonces? Entonces, junto a la más alta y eficiente sagacidad del cálculo que

\footnotetext{
${ }^{13}$ Paul Virilio, Velocidad y politica, op. cit., p. 131. Calificado como "profeta del horror" y tachado recurrentemente de apocalíptico, el filósofo postestructuralista ha recibido numerosas críticas por razón de su actitud pesimista y derrotista; véase, por ejemplo: Steve Redhead, Paul Virilio: Theorist for an accelerated culture, Toronto University Press, 2004. En relación al más que probable devenir autosuficiente de la tecnología, cabe recordar la aproximación panóptica desencadenada por los acontecimientos del 11-S, por la cual fue concebido un amplio abanico de tecnologías totalitarias, como las denominadas combat zones that see ("zonas de combate con visión propia") por el Pentágono, entre las que destacan cuatro millones de cámaras en las mayores urbes del planeta vinculadas a un único sistema en aras de alimentar los ordenadores de la base militar norteamericana, contrastando automáticamente múltiples rostros y vehículos en la base de datos donde se registran las personas "indeseables", como los sospechosos de terrorismo. Cfr. Michael Sniffen, Us develops urban surveillance system, Michael Sniffen, Us develops urban surveillance system, "Associated Press wire report", 1 (2003). En otro orden de cosas, la carrera por incrementar el poder de la implosión vehicular confirma la intuición fundamental de Lyotard sobre la condición postmoderna, la clausura irreversible de la concepción idealista (socrática) del saber y de los "metarrelatos" que constituyen su corolario, toda vez que la índole automática niega la estrategia, al capricho de la celeridad, como conocimiento previo y capacidad de razonamiento. Cfr. Jean-François Lyotard, La condición postmoderna: informe sobre el saber, Madrid, Cátedra, 2006.

${ }^{14}$ Cfr. Martin Heidegger, Serenidad, Barcelona, Serbal, 1989.
} 
planifica e inventa, coincidiría la indiferencia hacia el pensar (...), una total ausencia de pensamiento"15. Incapaz de controlar el progreso imparable de los nuevos medios de destrucción mediante estructuras de sentido distribuidas por canales morales y políticos, la "infocracia" contemporánea realiza en el plano óntico del poder fáctico, en efecto, los peores presagios del filósofo alemán, como reflejan los problemas existentes, cada vez más inapelables, a la hora de eliminar los "desechos" de la industria tecnológica" ${ }^{16}$.

\section{Epidemiología del nuevo malestar en la cultura}

\subsection{Etiología: La catástrofe temporal}

Somos conscientes de las múltiples posibilidades que brinda la evolución vertiginosa experimentada por la infoesfera. Como sostiene el propio Heidegger, sería miope condenar el mundo técnico como obra del diablo, pues todos dependemos de sus dispositivos en mayor o menor medida. Nos postramos, incluso, ante la inaudita capacidad de la racionalidad tecnocientífica para trascender todos los límites. Sin embargo, no podemos escapar al hecho de que la opresión tecnológica ejercida por la nueva dictadura del movimiento no sólo concierne a la esfera del poder, sino que también ha inundado amplias zonas de la existencia cotidiana. Como sostiene Virilio, "la carrera surge de la historia como una sublimación de la caza", la aceleración culmina el exterminio y la violencia de la velocidad, presentada más arriba como síntesis de control técnico e instrumental, se trueca simultáneamente en lugar y ley, sino y destino de la civilización, hasta el punto que todo ser humano tiene un proyecto con una ideología de aceleración perpetua ${ }^{17}$.

\footnotetext{
${ }^{15}$ Ibid., p. 29.

16 El documental "La tragedia electrónica" (Cosima Dannoritzer) denuncia que los países desarrollados generan anualmente 50 millones de toneladas de residuos electrónicos, lo cual representa un negocio mundial que mueve más dinero que el tráfico de drogas. En lugar de seguir el circuito oficial de reciclaje, un $75 \%$ de los desechos termina en vertederos ilegales de África y Asia, en beneficio de una red mafiosa de empresas fantasma que, alimentada por la codicia y la corrupción, atenta impunemente contra el medio ambiente y la salud pública.

${ }^{17}$ Además de Virilio, Harmut Rosa es seguramente el pensador que ha hecho mayor hincapié en la cuestión de la aceleración social que caracteriza a la Modernidad tardía. En Beschleunigung. Die Veränderung der Zeitstruckturen in der Moderne, el pensador canadiense elaboró, efectivamente, una consideración inicial sobre una teoría de la aceleración social que daba paso a distintos aspectos relacionados con ese tópico, como la modernidad y la aceleración, las distintas dimensiones de la aceleración social, sus efectos y manifestaciones (aceleración técnica, aceleración y cambio social y aceleración del ritmo de la vida), la aceleración como proceso autorreferente, las fuerzas motrices de la aceleración social, la relación entre la aceleración y el ejército, la identidad y la política situacionales, y la aceleración y petrificación como tentativa para redefinir la modernidad. Sin embargo, su principal aportación a la tesis de Virilio se encuentra delineada en Alienation as Acceleration. Towards a Critical Theory of Late-Modern Temporality. En esta obra, Rosa tiende un puente entre la aceleración y la alienación, definida como "vida mala", cuando trata de responder, a la luz de sus reflexiones sobre la
} 
Aunque no parece afectarnos de suyo en absoluto, lo cierto es que, bajo nuestro punto de vista, existe un vínculo inextricable entre la cadencia de las nuevas capacidades de los vectores y la extraordinaria proliferación de las llamadas "enfermedades de la adaptación", esos trastornos provocados por una reacción excesiva del organismo, obligado a movilizar sus defensas para atajar diversas agresiones, estados de tensión aguda o stress; en términos del propio Virilio: " $L a$ inmediatez de la información crea inmediatamente la crisis. (...) Imperceptible sobre el teclado de una computadora (...) desemboca en un encadenamiento catastrófico, ayer impensable. Con demasiada facilidad lo silenciamos, al lado del riesgo de proliferación ligado con las posibilidades nuevas de adquisición"18. Paradójicamente, el drama de nuestra época es que la misma ciencia que prolonga indefinidamente la esperanza de vida nos arroja a un torbellino de agitación cuyo ritmo vertiginoso no cesa de acelerar ${ }^{19}$, y lo que debería ser fuente de comodidad termina generando unas condiciones de vida que nos enferman, como manifiesta el nuevo malestar en la cultura que expande su influencia, silente pero lacerante, en la plenitud de su pujanza ${ }^{20}$. No se trata, empero, de un producto arbitrario pues, si Freud llevaba razón y la evolución de la cultura y del individuo son análogas, está justificado el diagnóstico de que una época e, incluso, la humanidad, se vuelva "neurótica" bajo la presión de ciertas ambiciones culturales ${ }^{21}$.

Desde el prisma de la psicopatología, las raíces del malestar preponderante se cifran, para nosotros, en el desfase patógeno, cacofonía o discrasia entre la velocidad absoluta del ciberespacio y el funcionamiento de los receptores

filosofía de Charles Taylor en Identität und kulturelle Praxis. Politische Philosophie nach, a la difícil interrogante de cómo vivir una "vida buena" en una sociedad que demanda un ritmo de vida acelerado y vertiginoso. Cfr. Juan Álvarez-Cienfuegos Fidalgo, La aceleración del tiempo como alienación. La propuesta de Hartmut Rosa desde la Teoría Crítica, "Acta Sociológica”, 69 (2016), p. 112.

${ }_{18}$ Paul Virilio, Velocidad y política, op. cit., pp. 127-128.

19 En la parte final del segundo capítulo de Alienation as acceleration, Rosa reconoce que los individuos son alcanzados constantemente por la trepidante rueda de la aceleración constante en su frenético impulso autónomo y descontrolado. A continuación, no obstante, llama la atención, frente a Virilio, sobre diversos fenómenos de desaceleración social, los límites naturales a la velocidad (el cuerpo humano y sus procesos), los oasis de desaceleración (islas remotas, los Amish, formas tradicionales de práctica social), procesos desencadenados por la propia aceleración (atascos de tráfico), formas intencionales de desaceleración (retiros a lugares de meditación o movimientos de la práctica slow), o movimientos ideológicos críticos con la aceleración (religiosos, ecologistas, ultraconservadores o anarquistas). Sin embargo, nuestra opinión es que, si bien cuestionan, aparentemente, el proceso de aceleración, se trata, en el fondo, de fenómenos complementarios inherentes al mismo, como ocurre con otras fuerzas de la Modernidad. Cfr. Juan Álvarez-Cienfuegos Fidalgo, op. cit., pp. 113-115.

${ }^{20}$ Más del $25 \%$ de la población sufre trastornos mentales y conductuales en algún momento. Afectan aproximadamente a un $10 \%$ de la población adulta. Alrededor del $20 \%$ de los pacientes atendidos por profesionales de atención primaria los padecen. En una de cada cuatro familias, cuentan con al menos uno de sus miembros entre sus víctimas. En 1990, eran responsables del 10\% de los AVAD (años de vida "sana" perdido) por enfermedad o lesión. En el año 2000, la tasa de prevalencia había ascendido al 12\%, y está previsto alcanzará el 15\% en 2020. Cfr. Luis Sáez Rueda, Enfermedades de Occidente. Patologías actuales del vacio desde el nexo entre filosofia y psicopatología [en Luis Sáez Rueda y otros, coords.: Occidente enfermo. Filosofia y patologías de civilización, Múnich, GRIN, 2011], p. 77.

${ }^{21}$ Cfr. Sigmund Freud, El malestar en la cultura, Madrid, Alianza, 2006, pp. 86-87. 
humanos, es decir, de sus cerebros y órganos sensoriales, por concurso del cual se trastorna el proceso comunicativo en perjuicio del vínculo entre conciencia y realidad ${ }^{22}$. Como sugieren diversos fenómenos de nuestra época como el cambio de contextos para valorar procesos globalmente, la apertura de ventanas de atención hipertextuales o la práctica del multitasking, el progreso ilimitado del infospace demanda una movilización permanente de nuestras facultades cognitivas y emotivas, de modo que los nuevos medios de masas vertebran, mediante la colaboración energética entre lo semiótico y lo estresante, un sistema sincronizado basado en el estrés a escala universal ${ }^{23}$.

Sin embargo, la materia física del organismo perceptivo y consciente posee límites naturales imborrables, por razón de los cuales necesita tiempos de elaboración racional y afectiva para traducir las reacciones inmediatas a través de la verbalización. En el lenguaje de la informática, los seres humanos y los nuevos medios de la destrucción estamos "formateados" siguiendo códigos diversos, de manera que los automatismos técnicos rebasan los ritmos funcionales del organismo hasta volverse independientes de la voluntad y la acción humanas. De hecho, si la atención se trata, como demuestra inequívocamente la ciencia económica, de un recurso escaso ${ }^{24}$, es porque la velocidad exhibida por los estímulos, mensajes, reclamos $\mathrm{y}$, sistemáticamente, todo intercambio comunicativo recorta drásticamente el tiempo mental necesario para valorarlos cabalmente y actuar en consecuencia; hablando en términos bélicos:

"Las velocidades supersónicas de los medios de asalto dejan (...) poco plazo a la detección, a la identificación y por tanto a la réplica. (...) La defensa activa exige por lo menos tener el tiempo material para intervenir. Pero es ese 'material de guerra' lo que desaparece en la aceleración de los rendimientos de los medios de comunicación de la destrucción. (...) Allí donde las deflagraciones

${ }^{22}$ Cfr. Franco Berardi, Generación post-alfa. Patologías e imaginarios en el semiocapitalismo, Buenos Aires, Tinta Limón, 2007, pp. 175-180. Podemos hablar de una "huelga de acontecimientos". Cfr. Macedonio Fernández, Papeles de Recienvenido y Continuación de la nada, Barcelona, Barataria, 2010 .

${ }^{23}$ Cfr. Peter Sloterdijk y Hans-Jürgen Heinrich, El sol y la muerte. Investigaciones dialógicas, Madrid, Siruela, 2004, pp. 78-89. En la línea de la Revolución Francesa, el universo cultural de la comunicación consuma la militarización de la existencia, satisfaciendo las expectativas creadas por Junger alrededor de la "guerra total". Hubo de pasar largo tiempo, en efecto, para entender su famosa misiva en El combate como una experiencia interior: "La guerra no es solamente nuestra madre, también es nuestra hija". Llegados a este punto, todos somos soldados del movimiento.

${ }^{24}$ Cfr. Thomas Davenport y John Beck, La economía de la atención: el nuevo valor de los negocios, Barcelona, Paidós, 2002. Marazzi denuncia las dificultades de los últimos operadores económicos a la hora de leer una página completa mediante un proceso secuencial o dedicar su atención al mismo objeto prolongadamente. Cfr. Christian Marazzi, Capitale e linguaggio. Dalla new economy all'economia di guerra, Roma, Derive Approdi, 2002. Por otro lado, la reducción de los tiempos de concentración alcanza su máxima expresión en la primera generación videoelectrónica, cuya configuración emotiva y cognitiva no responde tanto a relaciones entabladas con los otros como a la sumisa exposición a la semiosis de la máquina digital hipercompleja; en los Estados Unidos, por ejemplo, más de cinco millones de niños consumen diariamente el fármaco Ritalin para contrarrestar los denominados "trastornos de la atención". Franco Berardi, Generación post-alfa..., op. cit., p. 192. 
del explosivo (molecular o nuclear) contribuían a volver impropio para la existencia el espacio, de pronto son las del implosivo (vehículos vectores) los que reducen a nada el tiempo de actuar" 25 .

Es la "presentificación" correspondiente al tiempo real, la amputación del volumen del tiempo (de su profundidad de sentido) en detrimento de la multiplicidad de tiempos locales que comprendían la historia y la geografía hasta el advenimiento de un tiempo mundial único, un presente permanente que todo lo succiona, donde las experiencias y enseñanzas del pasado han perdido gran parte de su utilidad y no hay formas confiables de prever el futuro $^{26}$. Tras haber significado la superación de las distancias, la velocidad equivale repentinamente al abatimiento de la dimensión temporal, y los medios de comunicación de la ubicuidad desplazan a los medios de la historicidad. El énfasis del espíritu público sobre la domesticación del espacio en perjuicio del tiempo indica, no obstante, que los mass media han redistribuido las relaciones entre el aquí y el allá de forma más sensible que las relaciones entre el antes y el después: "Se ha subrayado repetidamente: nuestro territorio se amplía, nuestro calendario encoge; el horizonte óptico recula, la profundidad del tiempo se desdibuja y navegamos con mayor facilidad en la Web que en la cronología"27. De aquí se sigue una mutación cognitiva de la humanidad sin precedentes, una suerte de "hipercinesia interpretativa" donde la elaboración crítica de los estímulos que recibimos no sigue estrategias lineales de secuencia, sino procesos de "sobreinclusión" (overinclusion), espirales asociativas, conexiones asignificantes y transferencias rápidas que extienden los límites del significado hasta límites insospechados.

${ }^{25}$ Paul Virilio, Velocidad y politica, op. cit., pp. 122-123.

${ }^{26}$ Cfr. Paul Virilio y Philippe Petit, La politique du pire, París, Textuel, 1996, p. 79. Virilio describe la nueva noción de temporalidad, a contrapelo del tiempo que pasa de la historia y la cronología, como el tiempo que se expone instantáneamente; el tiempo de ese instante sin duración, un 'tiempo de exposición' (de sobreexposición o de infraexposición), cuya existencia habría estado prefigurada por las técnicas fotográficas y cinematográficas, el tiempo de un CONTINUUM privado de dimensiones físicas, donde el QUANTUM de acción (energética) y el PUNCTUM de observación (cinemática) se convertirían, súbitamente, en las últimas referencias de una realidad morfológica desaparecida, transferida al presente eterno de una relatividad cuyo espesor, profundidad topológica y teleológica serían las de este último instrumento de medida, esa velocidad de la luz que posee una dirección que es, al mismo tiempo, su magnitud y su dimensión y que se propaga a la misma velocidad en todas las direcciones...". Paul Virilio, L'espace critique, op. cit., p. 77.

Si contemplamos, en otro orden de cosas, la relación entre tiempo e infoesfera a la luz del método fenomenológico, tenemos que si bien el organismo consciente habita el tiempo, éste también está en el organismo consciente en cuanto duración de la conciencia (en el sentido de Bergson), más allá de la noción kantiana de tiempo como condición epistémica trascendental. Cfr. Paul Virilio, Esthetique de la disaparition. París, Galilée, 1988, p. 28.

${ }^{27}$ Régis Debray, Introducción a la mediología, Barcelona, Paidós, 2001, pp. 19-20. 


\subsection{Sintomatología: Expresiones mórbidas de superficie}

En contraste con la fijación moderna por la producción y la revolución, nuestro mundo histórico pone el acento, ciertamente, sobre la información y la comunicación. Ahora bien, los flujos de estimulaciones informativopublicitarias son un factor de hiper-excitación patógena del sistema emocional que generan con inusitada frecuencia patologías de sobrecarga excitante, entre las cuales destaca la afirmación impetuosa de la propia expresividad ${ }^{28}$. Como revela la moda de las radios libres, donde todos somos disc-jockeys, presentadores y animadores, no se trata de una simple ideología sino de un proceso de democratización sin parangón, un deseo compulsivo de la masa por expresar su propia intimidad para nada, sin otra finalidad que la mera expresión; es el placer de comunicar por comunicar, independientemente del "mensaje": "Eso es precisamente el narcisismo, la expresión gratuita, la primacía del acto de comunicación sobre la naturaleza de lo comunicado, la indiferencia por los contenidos, la reabsorción lúdica del sentido, la comunicación sin objetivo ni público, el emisor convertido en el principal receptor"29.

Como enseña Heidegger, el arte se define como la región privilegiada para el advenimiento de la verdad entendida, en un sentido fenomenológicoexistencial, como acontecimiento de sentido. Pues bien, el devenir contemporáneo de la actividad artística pone en evidencia, desde el art nouveau hasta el movimiento pop, que el proceso de "estetización del mundo" en curso, en virtud del cual todo cobra un sentido estético (incluso los objetos industriales de la vida cotidiana), hunde sus raíces en el fenómeno de la "hiperexpresión", a costa de la banalización irreversible de la obra de arte ${ }^{30}$. No obstante, la "hiperexpresión" descubre en la región del arte su convivencia con la lógica del vacío y la desusbstancialización postmoderna, toda vez que la hiperabundancia de realidad estetizada oculta una ausencia de realidad que desemboca en "desilusión estética": "El problema no es de pérdida de sentido,

${ }^{28}$ La consolidación del semiocapitalismo se traduce en la clínica psiquiátrica por un cambio de paradigma en la morbilidad de los cuadros nosológicos psiquiátricos más representativos desde la óptica de Freud. En contraposición a la neurosis, causada por la represión de la libido, las patologías dominantes presentan, como revela el estallido expresivo del just do it, un carácter esquizoide, asociado al exceso energético e informativo. Cfr. Franco Berardi, Generación post-alfa..., op. cit., pp. 211-223.

${ }^{29}$ Gilles Lipovetsky, op. cit., pp. 14-15.

${ }^{30}$ Caracterizado por Arthur Danto, a propósito de su deslumbrante aparición en la esfera artística neoyorquina, como "una especie de Midas, que vuelve todo lo que toca en el oro del arte puro", Andy Warhol constituye, a ojos vistas, un ejemplo paradigmático de nuestra perspectiva. A nuestro juicio, empero, el verdadero rey Midas del mundo del arte no es Warhol sino Marcel Duchamp. Y es que, a pesar de su aspecto novedoso, Warhol no hace más que prolongar la tradición artística occidental (a saber, la que asimila el valor del arte a su capacidad reproductiva), pues las elaboraciones de las "Cajas de Brillo" y de su homólogo industrial implican materiales y procesos diversos. En cambio, aunque sus primeros readymades eran collages de objetos, Duchamp no sólo imita objetos banales, sino que cambia de mundo un objeto de uso común para convertirlo en obra de arte. 
sino del demasiado sentido, del too much, de una proliferación del sentido, que a mi modo de ver afecta también al arte, afecta a la actividad artística. Hay una proliferación de expresión, de dar expresión a todo, de hacer que todo tenga un sentido estético. Eso para mí es la muerte del sentido, pero por exceso de sentido, y no por falta" ${ }^{1}$.

Junto a la "hiperexpresión", la hipermovilización nerviosa y el estrés informativo conllevan, cuando son frustrados, patologías de desinversión de la energía libidinal complementarias y simultáneas ${ }^{32}$. En primer lugar, tenemos la fatiga como síndrome paradigmático de nuestra era, consecuencia inevitable de una actividad desordenada que no alterna regularmente las fases de esfuerzo con el descanso obligatorio. Dado que desborda los ritmos funcionales del organismo, la velocidad exponencial de los instrumentos de comunicación representa, efectivamente, un factor de discordancia sumamente peligroso, provocando síntomas neurasténicos en función de los cuales nos mostramos incapaces de mantener el ritmo establecido. Generalmente, la fatiga se define como "disminución o pérdida de la excitabilidad de los músculos causada por exceso de excitaciones y de trabajo, que desemboca en deficiencia muscular acompañada de sensación especial de inercia"33. Ahora bien, los estados de agotamiento muscular no derivan necesariamente del esfuerzo físico dedicado a un trabajo efectivo, más allá de las averiguaciones de Mosso y During ${ }^{34}$. Además de la fatiga activa, resultado del desajuste entre una actividad determinada y nuestro ritmo fisiológico en función del tiempo disponible para llevarla a cabo, existe un agotamiento pasivo, inducido por una repetición hiperveloz de excitaciones sin la envergadura suficiente para contraer nuestros músculos per se, como ocurre en el régimen de la infocracia contemporánea.

La "hiperestimulación" que nos acecha fatiga nuestro organismo porque toda excitación dirigida sobre la extremidad receptora de un nervio de la sensibilidad obtiene resonancia por reflejo, como explica Etienne Grandjean,

${ }^{31}$ Jean Baudrillard, La ilusión y la desilusión estéticas, Caracas, Monte Ávila, 1997, p. 112. Sirvan como botón de muestra las consecuencias que tiene la aceleración del flujo de sílabas por segundo de la emisión vocal sobre la comprensión de los oyentes, en la medida que es inversamente proporcional al tiempo disponible para elaborar críticamente el mensaje y comprender su significado. Por lo demás, existen evidencias empíricas de que la "muerte del sentido" (Baudrillard) posee un alcance planetario, toda vez que la globalización conlleva ritmos de emisión cada vez más rápidos allí donde los regímenes autoritarios son sustituidos por los estilos de radio y teledifusión occidentales, como Oriente Medio, China o la Unión Soviética (la caída del comunismo tuvo como correlato una aceleración de la emisión de tres a seis sílabas por segundo). Cfr. Richard Robin, Russian-language listening comprehension: where are we going? where do we go? "Slavic and East European Journal", 35/3 (1991), pp. 403-410.

${ }^{32}$ Cfr. Pierre Vachet, Las enfermedades de la vida moderna, Barcelona, Labor, 1973.

33 Ibid., p. 17.

34 La neurofisiología moderna ofrece testimonios incuestionables sobre los mecanismos fundamentales de la fatiga, explicando fisiológicamente numerosos fenómenos que antaño parecían contradictorios. En resumen, el diencéfalo comprende estructuras nerviosas o centros de la fatiga que emiten influjos nerviosos a las regiones corticales, de manera tal que producen una sedación de las funciones físicas, sensoriales y psicomotoras. Cfr. Ibid., p. 21 
en los nervios motores del centro correspondiente y, como resultado, los músculos donde desembocan sufren un estado de inhibición general que oscila del tono fisiológico a la contracción muscular. Dicho proceso tiene lugar “automáticamente", pasa desapercibido para nuestro hipotálamo y, por ende, pensamos ingenuamente que somos máquinas inagotables, con una regulación ilimitada. El sistema global de comunicación se perfila, en cualquier caso, como el mejor caldo de cultivo para la "fatiga nerviosa", confirmando las sospechas levantadas en el simposio organizado por la Sociedad de Patología Comparada en la Facultad de Medicina de París (1959). Es el "mal del siglo", florece sin cesar y no existen barreras que la detengan.

Desde este punto de vista, pueden distinguirse cuatro grados de fatiga ${ }^{35}$ : "Decaimiento" (primeros síntomas), "agotamiento" (baja la tensión arterial, acelera el ritmo cardíaco y debilita los músculos), "surmenage" (irrita los centros nerviosos, impide el sueño, aumenta la tensión arterial y aminora el ritmo cardíaco) y "exceso" (puede parar bruscamente el corazón y favorecer síntomas transitorios de confusión mental por autointoxicación). Si bien el decaimiento conlleva predisposición al descanso, las condiciones de vida instituidas por el progreso tecnológico exigen que el sistema nervioso multiplique sus incitaciones motrices en la misma medida que los músculos se fatigan, de tal suerte que la sensación de malestar se torna cada vez más desagradable ante la imposibilidad de relajarnos. De hecho, la actividad del organismo desemboca a menudo en un estado crónico de fatiga, reconocible por una disminución de las actividades psicofisiológicas, síntomas psíquicos (irritabilidad, apatía, agresividad, ansiedad, depresión) y trastornos funcionales del sistema neurovegetativo (inapetencia, jaqueca, insomnio, trastornos circulatorios y digestivos, vértigo, palpitaciones, temblores, síncopes). Dado que no deriva del trabajo muscular, sensorial o intelectual sino del "esfuerzo de vivir" (Paul Chauchard), cobra sentido el carácter central de la fatiga a la altura del presente, síntoma común entre personas que no realizan, aparentemente, actividades análogas.

Por lo demás, existen evidencias empíricas de que la acumulación de fatiga recorta drásticamente la calidad de vida, pues repercute negativamente en las funciones físicas sensoriales, psicomotoras y nerviosas. A nivel fisiológico, la contracción muscular no sólo consume nuestras reservas de energía, sino que también libera en el interior de los tejidos desechos tóxicos que nuestro organismo encuentra muy difícil eliminar, desencadenando enfermedades del corazón, angina de pecho, nefritis, dolores reumáticos, úlceras de estómago e, incluso, muerte súbita, atribuible a una intoxicación bulbar por la toxina de la fatiga. En la medida que nos agota, el imperio de la velocidad absoluta también favorece la subida excesiva de presión sanguínea conocida como "hipertensión", uno de los misterios más oscuros de la fisiología contemporánea.

${ }^{35}$ Cfr. Ibid., p. 19.

Araucaria. Revista Iberoamericana de Filosofia, Política y Humanidades, año 19, no 38 . Segundo semestre de 2017. Pp. 49-71. ISSN 1575-6823 e-ISSN 2340-2199 doi: 10.12795/araucaria.2017.i38.03 
Aunque sabemos que deriva de una vasoconstricción, el porcentaje de casos con un origen contrastado empíricamente es prácticamente insignificante (del 10 al 20\%). Además, no existe consenso entre los cardiólogos sobre cuando cabe hablar de tensión anormal: Mientras la mayoría sostiene que la mínima y máxima presión varían, respectivamente, de 7 a 9 y de 12 a 14, la valoración de las personas de edad avanzada es objeto de mayores discrepancias. Sin embargo, resulta innegable que el factor nervioso juega un papel crucial al respecto, en tanto que los hipertensos padecen, a menudo, el síndrome de fatiga crónica conocido como "surmenage", término de origen francés para designar una depresión reactiva causada por agotamiento físico o emocional.

Según las últimas averiguaciones de la investigación anticancerosa, la aceleración del ritmo de vida favorece, para más escarnio, el desarrollo del cáncer, la enfermedad civilizatoria más temida ${ }^{36}$. Las características de la célula cancerosa son, a grandes rasgos, bien conocidas: Surge en un tejido determinado como mutación irreversible de células diversas morfológica y biológicamente, trastornando su almacenamiento de cromosomas de tal manera que multiplican su influencia anárquicamente hasta invadirlo todo, con menoscabo del equilibrio orgánico. Asimismo, sabemos que la aparición de un tumor maligno no es un proceso unívoco, sino que radica en tres factores inextricables: Genético, hormonal y provocador, que puede ser, a su vez, físico (rayos X, radio, rayos ultravioletas e infrarrojos), químico (colorantes, metales, extractos vegetales, grasas quemadas, suciedad atmosférica) o biológico (virus).

Pero independientemente de sus posibles causas, el factor psíquico y fisiológico ostenta una importancia inestimable en la evolución del cáncer, ya que puede acelerar, obstaculizar o, incluso, interrumpir su progresión, a priori, imparable. Las condiciones de extrema exigencia que sufrimos favorecen, es cierto, alteraciones físicas, químicas y hormonales que desembocan en trastornos vasomotores: Aceleración del pulso, sudor, temblor, espasmo y otras modificaciones en el interior de nuestras células. Por oposición a las emociones positivas que podrían contrarrestar la evolución del cáncer, empero, semejantes trastornos del sistema nervioso disminuyen la resistencia del organismo hasta propiciar eventualmente los desarreglos celulares responsables de su gestación y posterior desarrollo. En este sentido, el profesor Forgue señala que los trastornos funcionales neurovegetativos pueden intervenir fácilmente en el metabolismo sobre las glándulas endocrinas y sensibilizar a la célula al agente cancerígeno, toda vez que resultan, por razón de sus múltiples influencias, histológicamente incontrolables ${ }^{37}$.

${ }^{36}$ El cáncer representa un azote social sin parangón, y sus estragos no dejan de multiplicarse. Aproximadamente, causa una tercera parte de las muertes producidas allende los 45 años, y dos terceras partes después de los 65. En Francia, esta enfermedad quita la vida a más de 100.000 personas cada año. Cfr. Ibíd., p. 63.

${ }^{37}$ Cfr. Ibid., pp. 63-69. La radio constituye un ejemplo paradigmático de nuestro punto de vista. El 
Por otra parte, dado que muchos de ellos responden a un desequilibrio de los reflejos, existe un vínculo inextricable entre la "fatiga nerviosa" y los accidentes, en lo que constituye el tercer azote de la humanidad, tras las enfermedades cardiovasculares y el cáncer ${ }^{38}$. Como es sabido, la mayoría de gestos que realizamos ordinariamente no radican en nuestra voluntad sino en esos automatismos adquiridos inconscientemente que denominamos "reflejos". Cuando uno de nuestros sentidos es excitado, ciertas células recogen y transmiten la sensación correspondiente a la médula espinal, donde otra célula motriz trasfiere la orden de movimiento al músculo por una fibra nerviosa. Pues bien, según IvanoffSmolensky, la fatiga disminuye la velocidad de transmisión nerviosa una media de 30 metros por segundo, contribuyendo decisivamente en la multiplicación del número de accidentes y en el aumento de su gravedad. Por lo demás, el consumo generalizado de sustancias psicotrópicas, ya sea para ajustar el ritmo existencial a las exigencias comunicativas o bien para evadirse de ellas, no hace sino agravar el problema; por ejemplo, el alcohol aminora la rapidez de los reflejos del 15 al 25\%, mientras que los excitantes los aceleran del 20 al 100\% ${ }^{39}$.

A nivel psicológico, una de las expresiones morbosas de la fatiga más acuciante es la "depresión nerviosa", entendida como una flexión (transitoria o duradera) del equilibrio neuropsíquico que favorece la desgana, el pesimismo y el desinterés por la actividad habitual, esa patología de masas, cada vez más banalizada, del "están hartos" y del flip, entre otras expresiones de indiferencia y abandono. Asimismo, este Nihilismo new age, por así llamarlo, se traduce en numerosas patologías, como decaimiento, insomnio, adelgazamiento

Instituto Nacional de la Salud en Washington ha demostrado que las radiaciones electromagnéticas de gran longitud pueden afectar a las estructuras musculares complejas, como las de la célula viva. En el laboratorio de Bethesda (Maryland), un mono Rhesus (primate existente más parecido al hombre en términos fisiológicos) murió súbitamente tras verse sometido a ondas radioeléctricas con una frecuencia de 388 megaciclos y una potencia de 100 vatios. Bajo esta perspectiva, cabe preguntarse si la red de ondas hertzianas que constituye nuestro humus natural no tiene consecuencias en nuestro sistema nervioso y nuestro organismo. Cfr. Ibid., pp. 86-87.

${ }^{38}$ En Francia, los accidentes en carretera ocasionan anualmente 10.000 muertos y 25.000 inválidos. En Suiza, 200.000 accidentes causan de 300 a 400 muertes. De cada 10.000 trabajadores, una media de cinco muere por accidente laboral, y uno de cada ocho sufre al menos un accidente al año. En los Estados Unidos, el número de víctimas mortales por accidente laboral es de 14.000, sobre un total de 91.000 muertes por todo tipo de accidentes. En Francia, un millón de accidentes causan todos los años un paro de trabajo superior a 24 horas, entre los cuales 80.000 implican incapacidad de larga duración, permanente $\mathrm{o}$, incluso, la muerte. Más aún, el coste correspondiente para la colectividad supera los 400.000 millones de francos viejos, más de mil millones diarios; la seguridad social gasta en concepto de reparación e indemnización más de cien mil millones, y las empresas dedican 300.000 millones a solventar las pérdidas relativas a los paros de trabajo. Cfr. Ibid., pp. 87-88.

39 Tras el brote epidémico de polvos de los setenta y ochenta, algunos observan una epidemia de toxicomanía sin precedentes, alimentada por la proliferación de drogas legales que la industria farmacéutica pone a disposición de sus víctimas, antidepresivos, euforizantes y reguladores del humor como Ritalin, Prozac o Zoloft. Cfr. Franco Berardi, La fábrica de..., op. cit., pp. 23-24. No obstante, el consumo de alcohol constituye, a nuestros ojos, la tendencia evasiva más recurrente desde entonces; para comprobar con detalle la difusión del alcoholismo y sus estragos en la población: Cfr. Pierre Vachet, op. cit., pp. 110-118. 
considerable, lentitud en las funciones digestivas que acarrea anorexia, estreñimiento y un estado saburral acusado, ralentización de la respiración e hipotensión arterial, disminución o abolición de los reflejos tendinosos, temblor de fatiga, astenia acomodaticia de la visión, etc. En efecto, la tesis del "progreso" psicológico (E. Todd) es insostenible cuando cada vez son menos los que pueden escapar de los estados depresógenos ${ }^{40}$.

Como refleja la mirada sombría y la expresión melancólica de su rostro, el deprimido se caracteriza fundamentalmente por mantener un modo de vida inerte, abrumado por un sentimiento de inferioridad que lo colma de tristeza, ansiedad y una sensación de impotencia física por la que todos sus esfuerzos se centran en simples frivolidades, así como una incapacidad en el punto de realizar cualquier actividad intelectual, a tenor de la flexión de su atención y la lentitud evocadora de su memoria ${ }^{41}$. En este contexto, los problemas personales cobran dimensiones desmesuradas, y cuanto más insiste, al abrigo de los “psi”, menos los resuelve, de manera que el suicidio se vislumbra cada vez más como la única salida: “¿Qué cosa hoy no da lugar a dramatizaciones y stress? Envejecer, engordar, afearse, dormir, educar a los niños, irse de vacaciones, todo es un problema, las actividades elementales se han vuelto imposibles"42.

${ }^{40}$ El advenimiento de la sociedad de la información ha tenido como corolario un ascenso meteórico de la tasa de depresión en Europa Occidental y Norteamérica. Cfr. Gerald Klerman, Evidence for increase in rates of depression in North America and Western Europe in recent decades [en Hanns Hippius, Gerarld Klerman y Norbert Matussek, cords.: New results in depression research, Berlín, Springer, 1986], pp. 7-15; cfr. Gerald Klerman y Myma Weissman, Increasing rates of depression, "Journal of the American Medical Association", 261 (1989), pp. 2229-2235. Diversos estudios internacionales corroboran este dato en generaciones nacidas después de 1955. Cfr. Cross National Collaborative Group, The changing rate of major depression: Cross National comparisons, "Journal of the American Medical Association", 268 (1992), pp. 3098-3105. De acuerdo con la 55a Asamblea de la OMS, la depresión es actualmente la principal causa de discapacidad. Más de 120 millones de personas la padecen. Su tasa de prevalencia longitudinal es del 15\%, por lo que una de cada seis o siete personas sufre depresión en algún momento de su vida. En España es un problema de salud pública y afecta a poca menos población que en otros países de nuestro entorno, como Alemania (10\%), Francia (18\%), Reino Unido (17\%) o Irlanda (12\%). Cfr. Luis Sáez Rueda, Ficcionalización del mundo. Aportaciones para una crítica de patologías sociales, "Revista de Filosofia Universidad de Costa Rica", 45/115-116, 2007, p. 69. De cara al futuro, el panorama no puede ser más desalentador. Estudios recientes constatan que la tasa de prevalencia puntual y anual de enfermos depresivos presenta una curva epidemiológica ascendente en la población mayor de 15 años, los escolares y los pacientes asistidos en clínicas médicas generales o consultas psiquiátricas. El mismo equipo investigador ha confirmado esta impresión general mediante estudios de epidemiología comparativa apoyados en pruebas evaluadoras y criterios diagnósticos idénticos, dos exigencias difíciles de cumplir simultáneamente. Cfr. Francisco Alonso-Fernández, Factores determinantes en el incremento del trastorno depresivo en los últimos sesenta años [en Occidente enfermo..., op. cit.], p. 18.

${ }^{41}$ Los hábitos cotidianos del enfermo deprimido son especialmente sensibles a los males que amenazan a las personas que no realizan ejercicio físico de forma regular, como rigidez de las articulaciones y obesidad, definida como una sobrecarga ponderal del 20\%. En la actualidad, 205 millones de hombres y 300 millones de mujeres son obesos. Aunque existe la obesidad genética y endocrina, su influjo apenas puede compararse al de la obesidad exógena. Cfr. Ibíd., p. 56. Sin ir demasiado lejos, la televisión causa frecuentemente problemas característicos. Además de favorecer la obesidad, cuando contemplamos la pantalla adoptamos un estado de tensión nerviosa y contractibilidad, con el busto inclinado hacia delante, que produce una sensación de agujetas, así como una rigidez muscular en la zona de la nuca. Cfr. Pierre Vachet, op. cit., p. 86.

${ }^{42}$ Gilles, Lipovetsky, op. cit., p. 47. Dado que constituye el origen fundamental del $70 \%$ de los 


\section{Conclusión}

No nos resistimos a terminar nuestro trabajo sin rememorar la paradoja que atraviesa, como una flecha, el corazón del sistema de producción capitalista, esa contradicción interna formulada por Marx lapidariamente: "El Capitalismo produce sus propios sepultureros". Si prestamos atención a las consideraciones más elementales de su materialismo histórico, no podemos ignorar, empero, la singularidad irreductible de los diversos estadios que vertebran su evolución. Tendencialmente insaciable, la lógica acumulativa del Capital gravita desde siempre sobre el principio de la productividad. Pero mientras su fase industrial se basaba en extraer energía física de los trabajadores sin conceder importancia al sufrimiento psíquico concomitante, el Capitalismo tardío desplaza el acento sobre nuestra energía mental, precisamente lo que los nuevos medios de la destrucción están arruinando a marchas forzadas.

Efectivamente, el nuevo malestar en la cultura no puede seguir siendo marginalizado por los dueños del Capital, pues existe una estrecha relación entre la crisis de la new economy y la proliferación de la tristeza, la desmotivación y la depresión. Hasta cierto punto, la infelicidad constituye, es cierto, un estimulante del consumo, en tanto que comprar ayuda a calmar nuestra angustia. Sin embargo, el sufrimiento que muestran todos los índices epidemiológicos ha superado ampliamente el límite de lo soportable, razón por la cual tiene efectos de contracción del consumo. Baudrillard lo ha dicho todo al respecto: "Las cosas se están acelerando tanto que los procesos ya no se inscriben en una temporalidad lineal, en un despliegue lineal de la historia. Nada se mueve ya de la causa al efecto: todo se transversaliza por las inversiones del significado, por acontecimientos perversos, por inversiones irónicas. Aceleración, corrientes y turbulencias, autopotenciación y efectos caóticos" ${ }^{\prime 3}$.

En resumen, el sistema virtual de información alcanza extremos de sofisticación y rendimiento que podrían significar su disolución inminente. Esto es particularmente evidente en la infoesfera, donde el exceso de velocidad

suicidios consumados (apoyado por otros factores sociales y trastornos comórbidos), el crecimiento del porcentaje de suicidios en más del $60 \%$ constituye un índice epidemiológico indirecto del trastorno depresivo. Cfr. Francisco Alonso-Fernández, op. cit., pp. 21-23; cfr. George Murphy y Richard Wetzel: Suicide risk by birth cohort in the United States, 1949 to 1974, "Arch Gen Psychiatry", 37 (1980), pp. 519-523. Desde 1960 a 1980, este fenómeno ha sido registrado en cohortes sucesivas, con un intervalo de cinco años, según datos de la población sueca. Cfr. Lars Jacobsson y Ellinor Renberg, Epidemiology of Suicide in a Swedish country (Västerbotten), "Acta Psychiatr Scand", 74 (1986), pp. 459-468. En España, el incremento de la tasa de suicidio ha seguido una línea ascendente, alcanzando el $89,9 \%$ en la población masculina y el $115,9 \%$ en la población femenina durante los ochenta. $C f r$. José Manuel Gutiérrez García, El suicidio en España, 1981-1992, "Psicopatología", 16 (1995), pp. 51-53. En Italia, el acto suicida ha experimentado un aumentado significativo desde 1988 a 1994, con una cifra media de 10 suicidios por 100.000 habitantes, uno de los índices suicidas más bajo de los países europeos. Cfr. Eugenio Torre, Nunzia Chieppa y otros, Suicide and attempted in the province of Turin from 1988 to 1994: Epidemiological Analysis, "European Journal Psychiatry", 13 (1990), pp. 77-86.

${ }^{43}$ Jean Baudrillard, La ilusión vital, Buenos Aires, Siglo XXI, 2002, pp. 66-67. 
provoca una democratización sin precedentes de la "enfermedad de vivir", plaga difusa y endémica de nuestro tiempo. La desregulación del sistema se inscribe, no obstante, en un proceso de inversión más amplio: En la medida que avanza a la perfección, toda estructura (ya sea técnica o humana, económica, social o política) tiende a deconstruirse inexorablemente, por su propia sistematicidad, hasta implosionarlo todo. Es lo que Baudrillard denomina "ironía objetiva". Por eso, el imperio del monopolio no tiene más remedio que diseñar estrategias para contener nuestro ritmo de vida, moderar la infelicidad y estimular el consumo, siempre y cuando la humanidad no sea feliz porque, en ese caso, jamás comulgaría con la dictadura de la velocidad. 


\section{Referencias bibliográficas:}

Alan Sokal y Jean Brickmont, Imposturas intelectuales, Barcelona, Paidós, 1999. Christian Marazzi, Capitale e linguaggio. Dalla new economy all'economia di guerra, Roma, Derive Approdi, 2002.

Cross National Collaborative Group, The changing rate of major depression: Cross National comparisons, "Journal of the American Medical Association", 268 (1992).

Edwin Taylor y John Archibald Wheeler, Spacetime Physics, San Francisco, W. H. Freeman, 1966.

Eugenio Torre, Nunzia Chieppa y otros, Suicide and attempted in the province of Turin from 1988 to 1994: Epidemiological Analysis, "European Journal Psychiatry", 13 (1990).

Francisco Alonso-Fernández, Factores determinantes en el incremento del trastorno depresivo en los últimos sesenta años [en Luis Sáez Rueda y otros, coords.: Occidente enfermo. Filosofía y patologías de civilización, Múnich, GRIN, 2011].

Franco Berardi, La fábrica de la infelicidad. Nuevas formas de trabajo y movimiento global, Madrid, Traficantes de sueños, 2003.

George Murphy y Richard Wetzel: Suicide risk by birth cohort in the United States, 1949 to 1974, "Arch Gen Psychiatry", 37 (1980).

Gerald Klerman, Evidence for increase in rates of depression in North America and Western Europe in recent decades [en Hanns Hippius, Gerarld Klerman y Norbert Matussek, coords.: New results in depression research, Berlín, Springer, 1986].

Gerald Klerman y Myma Weissman, Increasing rates of depression, "Journal of the American Medical Association", 261 (1989).

Gilles Lipovetsky, La era del vacío. Ensayos sobre el individualismo contemporáneo, Barcelona, Anagrama, 1990.

Imanol Zubero, Los sindicatos españoles ante el cambio tecnológico (entre 1975 y 1990), Bilbao, Desclée de Brouwer, 1993.

Jean Baudrillard, La ilusión y la desilusión estéticas, Caracas, Monte Ávila, 1997. Jean Baudrillard, La ilusión vital, Buenos Aires, Siglo XXI, 2002.

Jean-François Lyotard, La condición postmoderna: informe sobre el saber, Madrid, Cátedra, 2006.

John Armitage, From modernism to hypermodernism and beyond: an interview with Paul Virilio [en Paul Virilio, From modernism to hypermodernism and beyond, Londres, Gran Bretaña, Sage, 2000].

José Manuel Gutiérrez García, El suicidio en España, 1981-1992, "Psicopatología", 16 (1995). 
Juan Álvarez-Cienfuegos Fidalgo, La aceleración del tiempo como alienación. La propuesta de Hartmut Rosa desde la Teoría Crítica, "Acta Sociológica", 69 (2016).

Lars Jacobsson y Ellinor Renberg, Epidemiology of Suicide in a Swedish country (Västerbotten), "Acta Psychiatr Scand”, 74 (1986).

Luis Sáez Rueda, Ficcionalización del mundo. Aportaciones para una crítica de patologías sociales, "Revista de Filosofía Universidad de Costa Rica", 45/115-116, 2007.

Luis Sáez Rueda, Enfermedades de Occidente. Patologías actuales del vacio desde el nexo entre filosofía y psicopatología [en Luis Sáez Rueda y otros, coords.: Occidente enfermo. Filosofía y patologías de civilización, Múnich, GRIN, 2011].

Martin Heidegger, Serenidad, Barcelona, Serbal, 1989.

Michael Sniffen, Us develops urban surveillance system, "Associated Press wire report", 1 (2003).

Paul Virilio, L'espace critique, París, Christian Bourgois, 1984.

Paul Virilio, Esthetique de la disaparition. París, Galilée, 1988.

Paul Virilio, Trans-Appearance, “Artforum”, 27, 10 (1989).

Paul Virilio, L'inertie polaire, París, Christian Bourgois, 1990.

Paul Virilio, Art of the motor, Minneapolis, University of Minnesota Press, 1995.

Paul Virilio, La vitesse de libération, París, Galilée, 1995.

Paul Virilio y Philippe Petit, La politique du pire, París, Textuel, 1996.

Paul Virilio, El cibermundo. La política de lo peor, Madrid, Cátedra, 1997.

Paul Virilio, Ground Zero, Londres, Verso, 2002.

Paul Virilio, Velocidad y política, Buenos Aires, La Marca, 2006.

Peter Sloterdijk y Hans-Jürgen Heinrich, El sol y la muerte. Investigaciones dialógicas, Madrid, Siruela, 2004.

Pierre Vachet, Las enfermedades de la vida moderna, Barcelona, Labor, 1973.

Pierre-Alain, Mercier, François Plassard y Víctor Scardigli, La sociedad digital, Barcelona, Ariel, 1985.

Ramón Zallo, La ciencia y la tecnología como relaciones sociales, "Telos", 17 (1989).

Régis Debray, Introducción a la mediología, Barcelona, Paidós, 2001.

Richard Robin, Russian-language listening comprehension: where are we going?

where do we go?, "Slavic and East European Journal", 35/3 (1991).

Steve Redhead, Paul Virilio: Theorist for an accelerated culture, Toronto University Press, 2004.

Thomas Davenport y John Beck, La economía de la atención: el nuevo valor de los negocios, Barcelona, Paidós, 2002.

Zygmunt Bauman, La società sotto assedio, Roma, Laterza, 2007. 
